\title{
WEAKLY COMPACT MULTILINEAR MAPPINGS
}

\author{
by RICHARD M. ARON* and PABLO GALINDO $\dagger$
}

(Received 8th June 1995)

\begin{abstract}
The notion of Arens regularity of a bilinear form on a Banach space $E$ is extended to continuous $m$-linear forms, in such a way that the natural associated linear mappings, $E \rightarrow \mathcal{L}\left({ }^{m-1} E\right)$ and $(m-1)$-linear mappings $E \times \ldots \times E \rightarrow E^{\prime}$, are all weakly compact. Among other applications, polynomials whose first derivative is weakly compact are characterized.
\end{abstract}

1991 Mathematics subject classification: Primary 46G20, Secondary 46B20

Let $E_{1}, \ldots, E_{m}$ be Banach spaces over scalar field $\mathbb{K}=\mathbb{R}$ or $\mathbb{C}$, and let $\mathcal{L}\left({ }^{m} E_{1}, \ldots, E_{m}\right)$ be the space of continuous $m$-linear forms $A: E_{1} \times \ldots \times E_{m} \rightarrow \mathbb{K}$. Each $A \in \mathcal{L}\left({ }^{m} E_{1}, \ldots, E_{m}\right)$ generates in a natural way $m$ linear mappings

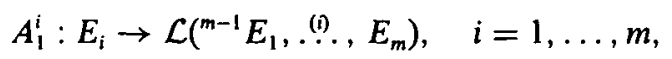

defined for $x \in E_{i}, x_{j} \in E_{j}, j=1, \ldots, m, j \neq i$ according to the formula

$$
A_{1}^{i}(x)\left(x_{1}, \ldots, x_{i-1}, x_{i+1}, \ldots, x_{m}\right)=A\left(x_{1}, \ldots, x_{i-1}, x, x_{i+1}, \ldots, x_{m}\right)
$$

as well as $m(m-1)$-linear mappings

$$
A_{m \cdot 1}^{i}: E_{1} \times \stackrel{(i)}{\stackrel{9}{1}} \times E_{m} \rightarrow E_{i}^{\prime}, \quad i=1, \ldots, m
$$

defined for $x \in E_{i}, x_{j} \in E_{j}, j=1, \ldots, m, j \neq i$ according to the formula

$$
A_{m-1}^{i}\left(x_{1}, \ldots, x_{i-1}, x_{i+1}, \ldots, x_{m}\right)(x)=A\left(x_{1}, \ldots, x_{i-1}, x, x_{i+1}, \ldots, x_{m}\right) \text {. }
$$

Note that whenever $E_{i}=E$ for all $i=1, \ldots, m$ and $A$ is symmetric, all mappings $A_{1}^{i}$ (resp. $A_{m-1}^{i}$ ) coincide.

R. Arens [2] (see also Ülger [18]) was among the first to recognize the relationship between extension to $E^{\prime \prime} \times F^{\prime \prime}$ of bilinear forms on $E \times F$ and properties of the associated linear mappings from $E$ to $F^{\prime}$. Specifically, a continuous bilinear $A$ form on

*Supported in part by NSF Grant Int-9023951.

† Supported in part by DGICYT pr. 91-0326 and by grant 93-081; the research of this author was undertaken during the academic year 1993-94 while visiting Kent State University to which thanks are given. 
$E \times F$ has a separately weak-* continuous extension to $E^{\prime \prime} \times F^{\prime \prime}$ if and only if the linear mapping $A_{1}^{1}$ (and hence $A_{1}^{2}$ ) from $E \rightarrow F^{\prime}$ is weakly compact. (This property has also been studied in recent years by Saab and Saab [16] among others.) This property, also called Arens regularity, was exploited in [6] to examine the homomorphisms on the Banach algebra $H^{\infty}\left(\dot{U}_{E}\right)$, where $U_{E}$ is the closed unit ball of $E$, as well as in [8] in connection with the Fréchet algebra $H_{b}(E)$ of all holomorphic functions on $E$ which are bounded on balls. (We refer to [11] for general background on holomorphic functions on infinite dimensional spaces, and to [13] for background on locally convex topologies.)

Here, we study the following generalized form of Arens regularity: An $m$-linear mapping $A \in \mathcal{L}\left({ }^{m} E_{1}, \ldots, E_{m}\right)$ is said to be regular if every mapping $A_{1}^{i}(i=1, \ldots, m)$ is weakly compact. (Recall that a mapping $g$ is said to be weakly compact if every point in the domain of $g$ has a neighbourhood with relatively weakly compact image.) If every $A \in \mathcal{L}\left({ }^{m} E\right)$ is regular, $E$ is said to be m-regular. In this note, we study this property, obtaining several equivalent conditions as well as some applications to function theory on $E$. Among other things, we will obtain a non-linear version of the factorization theorem of Davis, Figiel, Johnson and Pełczyniski ([10, Lemma 3 p. 227]) for $m$-regular mappings.

In our discussion, we will have frequent occasion to use the notional convention $\stackrel{(i)}{(i)}$ to mean that the $i$ th coordinate is not involved. For example, we have already employed this by writing $\mathcal{L}\left({ }^{m-1} E_{1},{ }^{(i)}, E_{m}\right)$ to denote $\mathcal{L}\left({ }^{m-1} E_{1}, \ldots, E_{i-1}, E_{i+1}, \ldots, E_{m}\right)$.

It is trivial that every reflexive space is $m$-regular for every $m \in \mathbb{N}$. There are also examples of non-reflexive $m$-regular spaces for every $m \in \mathbb{N}$ : By a classical result of Pełcyzǹski (see [14]) for every $m \in \mathbb{N}$, every $m$-linear form $A$ on $c_{0}$ is weakly continuous (on bounded sets). Then one can prove, in an analogous way to that used in [9] for symmetric multilinear forms, that $A_{1}^{i}$ is compact for all $i=1, \ldots, m$. Another (nonreflexive) example of an $m$-regular space for every $m \in \mathbb{N}$ is the James space, $T_{J}^{\prime}$, modelled on the original Tsirelson's space $T^{\prime}$ [7]. In fact, every $A \in \mathcal{L}\left({ }^{m} T_{j}^{\prime}\right)$ is weakly continuous on bounded sets: Indeed, no spreading model built on a normalised weakly null sequence in $T_{J}^{\prime}$ has a lower $q$-estimate for any $q<\infty$ [7]. Then the arguments of Farmer ([12, 1.3 Thm.]) show that every multilinear form on $T_{J}^{\prime}$ is weakly sequentially continuous at the origin and a standard induction argument leads to weak sequential continuity at any point. Since $T_{j}^{\prime}$ does not contain copies of $\ell_{1}$, it follows that every multilinear form on $T_{J}^{\prime}$ is weakly continuous on bounded sets. Therefore, as in the case of $c_{0}, A_{1}^{i}$ is compact for all $i=1, \ldots, m$.

There are also non-regular spaces, $\ell_{1}$ being the typical example ([6, p. 83]). Nevertheless, there are non-trivial regular bilinear forms on $\ell_{1}$, such as $A(x, y)=\sum x_{j} y_{j}$, where $x=\left(x_{j}\right)$ and $y=\left(y_{j}\right)$ are in $\ell_{1}$.

If $E$ is $m$-regular for some $m>2$, then $E$ is $(m-1)$-regular, and hence $E$ is regular. This will follow easily from the next theorem. To see this, let $A \in \mathcal{L}\left({ }^{m-1} E\right)$. Pick $\phi \in E^{\prime}$ and $z \in E$ such that $\phi(z)=1$. Since $B\left(x_{1}, \ldots, x_{m}\right)=A\left(x_{1}, \ldots, x_{m-1}\right) \phi\left(x_{m}\right)$ defines an element $B \in \mathcal{L}\left({ }^{m} E\right)$ which is regular by assumption, $B$ satisfies condition (5) in Theorem 1. Thus $A\left(x_{1}, \ldots, x_{m-1}\right)=B\left(x_{1}, \ldots, x_{m-1}, z\right)$ also fulfills that condition. However, the converse is false, as the following example in [5] (see also [8]) shows. Let 
$A: L_{\infty}[0,1] \times L_{\infty}[0,1] \times L_{\infty}[0,1] \rightarrow \mathbb{C}$ be defined as

$$
A(f, g, h)=\sum_{j=1}^{\infty}\left(2^{j} \int_{\left[\frac{1}{2}, \frac{1}{2 j-1}\right]} f d x\right) \int_{[0,1]} g r_{j} d x \int_{[0,1]} h r_{j} d x
$$

where $r_{j}$ is the standard $j$ th Rademacher function. Since $A$ is not regular, $L_{\infty}[0,1]$ is not 3-regular; of course, $L_{\infty}[0,1]$ is regular since it is a $C^{*}$-algebra. See [5] for details.

The following argument shows that for every $m \geq 2$, the space $c_{0} \times \ell_{m}$ is $m$-regular but not $(m+1)$-regular. Since the dual of $c_{0} \times \ell_{m}$ is $\ell_{1} \times \ell_{m / m-1}$, it suffices to show that every $A \in \mathcal{L}\left({ }^{m-1} c_{0} \times \ell_{m}, \ell_{1}\right)$ is weakly compact. That $A$ is weakly sequentially continuous follows from $\left(\left[1,3.2\right.\right.$ Cor. 5]) once one realizes that $c_{0} \times \ell_{m}$ has property $P_{1 / m}$ (see [1] for details). Moreover, by ( $[9$, Cor. 2.5]) $A$ maps weakly Cauchy sequences into norm convergent ones and therefore $A$ is compact because neither $c_{0}$ nor $\ell_{m}$ contains copies of $\ell_{1}$. So $c_{0} \times \ell_{m}$ is $m$-regular. On the other hand, since the $m$-linear mapping $\Pi: \ell_{m} \times \ldots \times \ell_{m} \rightarrow \ell_{1}$ given by $\Pi\left(\left(x_{1}^{i}\right), \ldots,\left(x_{m}^{i}\right)\right)=\left(x_{1}^{i} \cdots x_{m}^{i}\right)_{i}$ is not weakly compact, it follows that $c_{0} \times \ell_{m}$ is not $(m+1)$-regular.

The following result gives a reasonably useful characterization of $m$-regularity. Note that $E_{1} \hat{\otimes} E_{2}$ denotes the completed projective tensor product of $E_{1}$ and $E_{2}$.

Theorem 1. Let $A \in \mathcal{L}\left({ }^{m} E_{1}, \ldots, E_{m}\right)$. Then the following are equivalent:

(1) $A$ is regular, i.e., $A_{1}^{i}: E_{i} \rightarrow \mathcal{L}\left({ }^{m-1} E_{1}, . \stackrel{(i)}{.}, E_{m}\right)$ is a weakly compact linear operator for all $i=1, \ldots, m$.

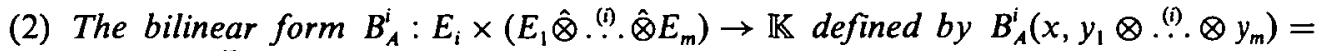
$A\left(y_{1}, \ldots, \stackrel{(i)}{x}, \ldots, y_{m}\right)$ is Arens regular for all $i=1, \ldots, m$.

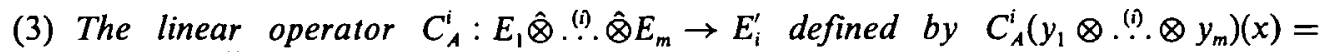
$A\left(y_{1}, \ldots, \stackrel{(i)}{x}, \ldots, y_{m}\right) \quad\left(x \in E_{i}\right)$ is weakly compact for all $i=1, \ldots, m$.

(4) The mapping $A_{m-1}^{i}: E_{1} \times \stackrel{(i)}{.} \times E_{m} \rightarrow E_{i}^{\prime}$ is weakly compact for all $i=1, \ldots, m$.

(5) For each $i=1, \ldots, m$, there is a weakly compact subset $L_{i}$ of $E_{i}^{\prime}$ such that

$$
\left|A\left(x_{1}, \ldots, x_{m}\right)\right| \leq\left\|x_{1}\right\|_{L_{1}} \ldots\left\|x_{m}\right\|_{L_{m}}
$$

for all $x_{1} \in E_{1}, \ldots, x_{m} \in E_{m}$.

(6) For each $i=1, \ldots, m$, there is a reflexive Banach space $Y_{i}$, a continuous linear mapping $T_{i}: E_{i} \rightarrow Y_{i}$ and an m-linear mapping $D \in \mathcal{L}\left({ }^{m} Y_{1}, \ldots, Y_{m}\right)$ such that

$$
A\left(x_{1}, \ldots, x_{m}\right)=D\left(T_{1}\left(x_{1}\right), \ldots, T_{m}\left(x_{m}\right)\right)
$$

for all $x_{1} \in E_{1}, \ldots, x_{m} \in E_{m}$. 


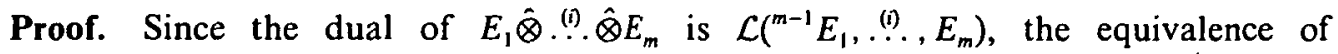
conditions (1), (2) and (3) follows from ([18, Th. 2.2]) applied to each $B_{A}^{i}$ for all $i=1, \ldots, m$. That (3) implies (4) is immediate and the proof of (4) $\Rightarrow$ (3) follows easily since the closed unit ball of $E_{1} \hat{\otimes} \stackrel{(i)}{\stackrel{\otimes}{\otimes} E_{m}}$ is the closure of the absolutely convex hull of $U_{E_{1}} \otimes \stackrel{(i)}{\stackrel{9}{*}} \otimes U_{E_{m}}$.

(4) $\Rightarrow$ (5). Set $K_{i}=A_{m-1}^{i}\left(U_{E_{1}} \times \stackrel{(i)}{.} \times U_{E_{m}}\right) . K_{i}$ is a relatively weakly compact set in $E_{i}^{\prime}$. Moreover, given $x \in E_{i}$ and $u_{1} \in E_{1}, \stackrel{(n)}{.}, u_{m} \in E_{m}$, all of norm $\leq 1$, we have $\left|A\left(u_{1}, \ldots, \stackrel{(i)}{x}, \ldots, u_{m}\right)\right|=\left|A_{m-1}^{i}\left(u_{1}, \ldots, u_{m}\right)(x)\right| \leq \sup _{x^{\prime} \in K_{i}}\left|\left\langle x^{\prime}, x\right\rangle\right|=\|x\|_{K_{i}}$ for all $i=1, \ldots, m$. Consequently $\mid A\left(u_{1}, \ldots,\left(\stackrel{())}{x}, \ldots, u_{m}\right) \mid \leq\|x\|_{K_{i}}\left\|u_{1}\right\| . \stackrel{(i)}{.}\left\|u_{m}\right\|\right.$ for all $i=1, \ldots, m$. By enlarging $K_{i}$ we may assume that $K_{i}$ is absolutely convex and weakly compact. Therefore if $\left\|u_{k}\right\| \leq j$ for all $k \neq i$, we have

$$
\left|A\left(u_{1}, \ldots, \stackrel{(i)}{x}, \ldots, u_{m}\right)\right| \leq j^{m-1}\|x\|_{K_{i}} \text { for all } i=1, \ldots, m
$$

We denote by $M^{\circ}$ the polar of any set $M$ in the dual pair $\left(E_{i}^{\prime}, E_{i}\right)$ and by $\Gamma(M)$ the absolutely convex hull of $M$. Consider

$$
L_{i}=\bigcap_{j=1}^{\infty}\left(j^{m-1} K_{i} \cup \frac{U_{E_{i}^{\prime}}}{j}\right)^{o o}
$$

We apply Grothendieck's lemma ([10, Lemma 2 p. 227]) to show that $L_{i} \subset E_{i}^{\prime}$ is weakly compact. Indeed, given $\epsilon>0$ and $j \in \mathbb{N}$ such that $1<j \epsilon$, we have $L_{i} \subset\left(j^{m-1} K_{i} \cup\right.$ $\left.U_{E_{i}^{\prime}} / j\right)^{o o}=c l_{w\left(E_{i}^{\prime}, E_{i}\right)} \Gamma\left(j^{m-1} K_{i} \cup U_{E_{i}^{\prime}} / j\right) \subset c l_{w\left(E_{i}^{\prime}, E_{i}\right)}\left(j^{m-1} K_{i}+U_{E_{i}^{\prime}} / j\right)=\left(j^{m-1} K_{i}+U_{E_{i}^{\prime}} / j\right)$, the last equality being true because the sum of two weak* compact sets is weak* closed.

Now,

$$
L_{i}^{o}=c l_{\left.m E_{i}, E_{i}^{\prime}\right)} \Gamma\left\{\cup_{j=1}^{\infty}\left(j^{m-1} K_{i} \cup \frac{U_{E_{i}^{\prime}}}{j}\right)^{o}\right\}=c l_{\|\|_{E_{i}}} \Gamma\left\{\cup_{j=1}^{\infty}\left(j^{m-1} K_{i} \cup \frac{U_{E_{i}^{\prime}}}{j}\right)^{o}\right\}
$$

the last equality being true because of the convexity of $\Gamma\left\{\cup_{j=1}^{\infty}\left(j^{m-1} K_{i} \cup U_{E_{i}^{\prime}} / j\right)^{\circ}\right\}$.

Suppose $x_{i} \in L_{i}^{o}$ for all $i=1, \ldots, m$. We will show that $\left|A\left(x_{1}, \ldots, x_{m}\right)\right| \leq 1$. Since $A$ is continuous, it suffices to prove that $\left|A\left(x_{1}, \ldots, x_{m}\right)\right| \leq 1$ just for $x_{i} \in \Gamma\left(\cup_{j=1}^{\infty}\left(j^{m-1} K_{i} \cup\right.\right.$ $\left.\left.U_{E_{i}^{\prime}} / j\right)^{o}\right\}, i=1, \ldots, m$. Adding zeros if necessary, we may suppose without loss of generality that $x_{i}=\sum_{j=1}^{n} \alpha_{i, j} y_{i, j}$ where $y_{i, j} \in\left(j^{m-1} K_{i} \cup U_{E_{i}^{\prime}} / j\right)^{\circ}$ and $\sum_{j=1}^{n}\left|\alpha_{i, j}\right| \leq 1$ for all $i=1, \ldots, m$. Then

$$
\begin{aligned}
& A\left(x_{1}, \ldots, x_{m}\right)=A\left(\sum_{j_{1}=1}^{n} \alpha_{1, j_{1}} y_{1, j_{1}}, \ldots, \sum_{j_{m}=1}^{n} \alpha_{m, j_{m}} y_{m, j_{m}}\right)= \\
& \sum_{j_{1}=1}^{n} \ldots \sum_{j_{m}=1}^{n} \alpha_{1, j_{1}} \ldots \alpha_{m, j_{m}} A\left(y_{1, j_{1}}, \ldots, y_{m, j_{m}}\right)
\end{aligned}
$$


so it is enough to check that $\left|A\left(y_{1, j_{1}}, \ldots, y_{m, j_{m}}\right)\right| \leq 1$ for any choice of $\left(1, j_{1}\right), \ldots,\left(m, j_{m}\right)$. Since $\left\|y_{i, j}\right\| \leq j$ for all $i$ and $j$, we may assume without loss of generality that $\left\|y_{i, j_{i}}\right\| \leq j_{m}$ for all $i=1, \ldots, m$, and so $\left|A\left(y_{1, j_{1}}, \ldots, y_{m, j_{m}}\right)\right| \leq\left(j_{m}\right)^{m-1}\left\|y_{m, j_{m}}\right\|_{K} \leq 1$ because $y_{m, j_{m}} \in\left(j_{m}^{m-1} K_{m}\right)^{o}$.

$(5) \Rightarrow(4)$. We may suppose that $L_{i}$ is also absolutely convex for all $i=1, \ldots, m$. Since the $L_{i}$ 's are weakly compact, there is a constant $\lambda>0$ such that for all $i, \frac{1}{2} L_{i}$ is contained in the unit ball of $E_{i}^{\prime}$, i.e. $\|x\|_{L_{i}} \leq \lambda\|x\|$ for all $x \in E_{i}$. We will check that, for any $i=1, \ldots, m$, the set $A_{m-1}^{i}\left(U_{E_{1}} \times \stackrel{(I)}{\cdots} \times U_{E_{m}}\right)$ is relatively weakly compact by proving that $A_{m-1}^{i}\left(U_{E_{1}} \times \stackrel{(i)}{?} \times U_{E_{m}}\right) \subset \lambda^{m-1} L_{i}$. Let $z \in E_{i}^{\prime \prime}$ belong to the polar set of $L_{i}$ with respect to the dual pair $\left(E_{i}^{\prime}, E_{i}^{\prime \prime}\right)$ and let $\left\{x_{k}\right\}_{k \in \Lambda}$ be a net in $E_{i}$ converging to $z$ in the Mackey topology $\mu\left(E_{i}^{\prime \prime}, E_{i}^{\prime}\right)$ (such a net exists because $E_{i}$ is a $w\left(E_{i}^{\prime \prime}, E_{i}^{\prime}\right)$-dense convex subset which is of the same dual pair as the $\mu\left(E_{i}^{\prime \prime}, E_{i}^{\prime}\right)$-topology) $([13,8.2 .5])$. Now if $u_{1} \in U_{E_{1}}, \stackrel{(i)}{.}, u_{m} \in U_{E_{m}}$ we have

$$
\left\langle A_{m-1}^{i}\left(u_{1}, \ldots, u_{m}\right), z\right\rangle=\lim _{k}\left\langle A_{m-1}^{i}\left(u_{1}, \ldots, u_{m}\right), x_{k}\right\rangle=\lim _{k} A\left(u_{1}, \ldots, \stackrel{(1)}{x}_{k}, \ldots, u_{m}\right) .
$$

Therefore, since $\left|A\left(u_{1}, \ldots, \stackrel{(i)}{x_{k}}, \ldots, u_{m-1}\right)\right| \leq\left\|u_{1}\right\|_{L_{1}} \ldots\left\|x_{k}^{(i)}\right\|_{L_{i}} \ldots\left\|u_{m}\right\|_{L_{m}} \leq \lambda^{m-1}\left\|x_{k}\right\|_{L_{i}}$ and $\lim _{k}\left\|x_{k}\right\|_{L_{i}}=\|z\|_{L_{i}} \leq 1$, we have $\left|\left\langle A_{m-1}^{i}\left(u_{1}, \stackrel{(i)}{.}, u_{m}\right), z\right\rangle\right| \leq \lambda^{m-1}$.

Thus according to the bipolar theorem we have proved that $A_{m-1}^{i}\left(U_{E_{1}} \times \stackrel{(i)}{\cdots} \times U_{E_{m}}\right)$ is contained in the $w\left(E_{i}^{\prime}, E_{i}^{\prime \prime}\right)$-closure of $\lambda^{m-1} L_{i}$, which is itself.

$(5) \Rightarrow(6)$. The next part of the proof relies on the following: If for some absolutely convex closed set $L \subset X$ the Banach space $X_{L}$ generated by $L$ is reflexive and if ${ }^{\bullet}$ denotes the polar in the dual pair $\left\langle X^{\prime}, X^{\prime \prime}\right\rangle$, then the completion of $X_{\left(L^{\circ}\right)}^{\prime}=\left(X^{\prime} /\|\cdot\|_{L}^{-1}(0),\|\cdot\|_{L}\right)$ is a reflexive Banach space. To see this, observe that since $L$ is weakly closed and absolutely convex, we have $L^{* *}=L$ and $X_{L}^{\prime \prime}=X_{L}$. Moreover, $\left(X^{\prime} /\|\cdot\|_{L}^{-1}(0),\|\cdot\|_{L}\right)^{\prime}=X_{L}^{\prime \prime}$. (see [13, 8.3.4]), so it follows that the dual of the completion of $X_{(L \cdot)}^{\prime}$ is reflexive. Hence the completion of $X_{\left(L^{\circ}\right)}^{\prime}$, which we denote as usual by $\widetilde{\left.X_{\left(L^{\circ}\right)}\right)}$, is reflexive.

The assumption on $A$ guarantees that $A$ is uniformly continuous on bounded subsets of $E_{1} \times \ldots \times E_{m}$ when each space $E_{i}$ is endowed with the topology of uniform convergence on weakly compact sets in $E_{i}^{\prime}$. Moreover, since the points in the bidual are in the weak*-closure of multiples of the unit ball and the $\mu\left(E_{i}^{\prime \prime}, E_{i}^{\prime}\right)$-closure of any convex set coincides with its $w\left(E_{i}^{\prime \prime}, E_{i}^{\prime}\right)$-closure, it follows that every point in $\left(E_{1}^{\prime \prime}, \mu\left(E_{1}^{\prime \prime}, E_{1}^{\prime}\right)\right) \times \ldots \times\left(E_{m}^{\prime \prime}, \mu\left(E_{m}^{\prime \prime}, E_{m}^{\prime}\right)\right)$ is the limit of a bounded net in $E_{1} \times \ldots \times E_{m}$. Therefore $A$ can be uniquely extended to $\left(E_{1}^{\prime \prime}, \mu\left(E_{1}^{\prime \prime}, E_{1}^{\prime}\right)\right) \times \ldots \times\left(E_{m}^{\prime \prime}, \mu\left(E_{m}^{\prime \prime}, E_{m}^{\prime}\right)\right)$ by uniform continuity on bounded sets. Let $\hat{A}$ be this extension. It is easy to check that $\hat{A}$ is $m$-linear and that the estimates in (5) are preserved, i.e.,

$$
\left|\hat{A}\left(x_{1}^{\prime \prime}, \ldots, x_{m}^{\prime \prime}\right)\right| \leq\left\|x_{1}^{\prime \prime}\right\|_{L_{1}} \ldots\left\|x_{m}^{\prime \prime}\right\|_{L_{m}} .
$$

Let $q_{i}:\left(E_{i}^{\prime \prime}, \mu\left(E_{i}^{\prime \prime}, E_{i}^{\prime}\right)\right) \rightarrow\left(E_{i}^{\prime \prime} /\|\cdot\|_{L_{i}}^{-1}(0),\|\cdot\|_{L_{i}}\right)$ be the canonical quotient mapping. It is a routine calculation to verify that the mapping 


$$
\hat{\hat{A}}:\left(E_{1}^{\prime \prime} /\|\cdot\|_{L_{1}}^{-1}(0),\|\cdot\|_{L_{1}}\right) \times \ldots \times\left(E_{m}^{\prime \prime} /\|\cdot\|_{L_{m}}^{-1}(0),\|\cdot\|_{L_{m}}\right) \rightarrow \mathbb{K}
$$

defined by

$$
\hat{\hat{A}}\left(q_{1}\left(x_{1}^{\prime \prime}\right), \ldots, q_{m}\left(x_{m}^{\prime \prime}\right)\right)=\hat{A}\left(x_{1}^{\prime \prime}, \ldots, x_{m}^{\prime \prime}\right)
$$

is well-defined, $m$-linear and continuous. Once again, $\hat{\hat{A}}$ is uniformly continuous on bounded sets, and hence it can be extended to a mapping $D$ on the product of the completions of $\left(E_{i}^{\prime \prime} /\|\cdot\|_{L_{i}}^{-1}(0),\|\cdot\|_{L_{i}}\right)$.

By the classical result of Davis, Figiel, Johnson and Pełczyǹski ([10, Lemma 3 p. 227]), we may choose the weakly compact sets $L_{i}$ such that the Banach spaces $\left(E_{i}^{\prime}\right)_{L_{i}}$ are reflexive and the $L_{i}$ 's are absolutely convex. To complete the argument, it suffices to apply the observation stated above.

(6) $\Rightarrow$ (5). $\quad\left|A\left(x_{1}, \ldots, x_{m}\right)\right|=\left|D\left(T_{1}\left(x_{1}\right), \ldots, T_{m}\left(x_{m}\right)\right)\right| \leq\|D\| \cdot\left\|T_{1}\left(x_{1}\right)\right\| \ldots\left\|T_{m}\left(x_{m}\right)\right\| \quad$ and for each $i=1, \ldots, m, \quad\left\|T_{i}\left(x_{i}\right)\right\|=\sup _{y \in U_{Y_{i}^{\prime}}}\left|\left\langle y, T_{i}\left(x_{i}\right)\right\rangle=\sup _{y \in U_{Y_{i}^{\prime}}}\right|\left\langle T_{i}^{*} y, x_{i}\right\rangle \mid=\left\|x_{i}\right\|_{T_{i}^{*}\left(U_{r_{i}^{\prime}}\right)}$. Since $Y_{i}$ is reflexive, $T_{i}^{*}\left(U_{Y_{i}^{\prime}}\right)$ is a weakly compact set in $E_{i}^{\prime}$.

Theorem 1 yields the following factorization result.

Corollary 2. Let $P: E \rightarrow \mathbb{K}$ be a continuous m-homogeneous polynomial. If the derivative $d P: E \rightarrow E^{\prime}$ is weakly compact, then $P$ is continuous for the topology on $E$ of uniform convergence on weakly compact sets in $E^{\prime}$. Moreover there is a reflexive Banach space $Y$, a continuous linear mapping $T: E \rightarrow Y$ and a polynomial $Q: Y \rightarrow \mathbb{K}$ such that $P=Q \circ T$.

Proof. Let $A$ be the symmetric $m$-linear mapping associated to $P$. Since $d P(x)(u)=m A(u, x, \ldots, x)$ for $x, u \in E$, it follows that the unique $(m-1)$-linear symmetric form associated to the polynomial $d P: E \rightarrow E^{\prime}$ is $m A_{m-1}^{1}$. By the polarization formula (see e.g. $[11,1.1 .5]$ ) the weak compactness of $d P$ leads to the weak compactness of $m A_{m-1}^{1}$, so $A$ is regular. A glance at condition (5) in the above theorem suffices to prove the continuity statement. The factorization result follows from the fact that in this case all the spaces $Y_{i}$ constructed in the proof of $(5) \Rightarrow(6)$ coincide.

Corollary 3. (1) Let $A \in \mathcal{L}\left({ }^{m} E_{1}, \ldots, E_{m}\right)$. Then $A$ is weakly uniformly continuous on bounded subsets of $E_{1} \times \ldots \times E_{m}$ if and only if there are compact sets $C_{i} \subset E_{i}^{\prime}$ such that $\left|A\left(x_{1}, \ldots, x_{m}\right)\right| \leq\left\|x_{1}\right\|_{c_{1}} \ldots\left\|x_{m}\right\|_{c_{m}}$ for all $x_{1} \in E_{1}, \ldots, x_{m} \in E_{m}$.

(2) Let $P: E \rightarrow \mathbb{K}$ be a continuous m-homogeneous polynomial. Then $P$ is weakly uniformly continuous on bounded subsets of $E$ if and only if there is a compact set $C \subset E^{\prime}$ such that $|P(x)| \leq\|x\|_{c}^{m}$ for all $x \in E$.

The proof of this result, due originally to E. Toma [17], is a straightforward modification of the arguments used to prove Theorem 1. We note that the space of 
polynomials which are weakly uniformly continuous on bounded sets has proved useful in several situations (e.g. [6] and [9]).

The following will be useful in the proof of Theorem 5, below.

Remark 4. (1) If $A$ is the symmetric $m$-linear mapping associated to a polynomial $P: E \rightarrow \mathbb{K}$, then the sets $K_{i}$ considered in the proof of (4) $\Rightarrow(5)$ in Theorem 1 are actually $\Gamma\left(A_{m-1}^{1}\left(U_{E} \times \ldots \times U_{E}\right)\right)$, which is contained in $\left[(m-1)^{m-1} /(m-1) ! m\right] \Gamma\left(d P\left(U_{E}\right)\right)$.

(2) In the proof of $(4) \Rightarrow(5)$ in Theorem 1, replacing $K_{i}$ by $\alpha K_{i}$ leads to a weakly compact set $L_{i}(\alpha)=\bigcap_{j=1}^{\infty}\left(j^{m-1} \alpha K_{i} \cup U_{E_{i}^{\prime}} / j\right)^{o o}$ such that $\left|A\left(x_{1}, \ldots, x_{m}\right)\right| \leq \alpha$ if $x_{i} \in L_{i}(\alpha)^{o}$ for all $i=1, \ldots, m$.

Theorem 5. Suppose $E$ is a complex Banach space. Let $f \in H_{b}(E)$. The following are equivalent:

(1) $d f: E \rightarrow E^{\prime}$ is weakly compact.

(2) There is a reflexive Banach space $Y$, a continuous linear mapping $T: E \rightarrow Y$ and a holomorphic function $\hat{f} \in H_{b}(Y)$ such that $f=\hat{f} \circ T$.

(3) $f$ is continuous for the topology on $E$ of uniform convergence on weakly compact sets in $E^{\prime}$.

(4) $f$ is uniformly continuous on bounded sets for the topology on $E$ of uniform convergence on weakly compact sets in $E^{\prime}$.

Proof. (1) $\Rightarrow(2)$. First of all, observe that $d f$ is also of bounded type. For, by Cauchy's inequality, $\|d f(x)\|=\sup _{u \in U_{E}}|d f(x)(u)| \leq \sup _{u \in U_{E}}|f(x+u)|$ for all $x \in E$. Arguing as in Thm. 3.2 of [15], it follows that $d f$ maps bounded sets into relatively weakly compact sets in $E^{\prime}$.

Let $\sum P_{m}$ be the Taylor series expansion of $f$ around 0 and recall that $\sum d P_{m}$ is the Taylor series expansion of $d f$ around 0. Applying Thm. 3.2 of [15], it follows that $d P_{m}$ is weakly compact for all $m \in \mathbb{N}$. For each $P_{m}$ we construct the weakly compact set $L_{m}=\bigcap_{j=1}^{\infty}\left(j^{m-1} c l_{w\left(E, E^{\prime}\right)}\left\{\Gamma\left(d P_{m}\left(U_{E}\right)\right\} \cup U_{E^{\prime}} / j\right)\right)^{o o}$. As we mentioned in Remark 4, if $x \in L_{m}^{o}$, $\left|P_{m}(x)\right| \leq\left[(m-1)^{m-1} /(m-1) ! m\right]$. On the other hand, for any $m, j \in \mathbb{N}$, we have $j^{m-1} \Gamma\left\{d P_{m}\left(U_{E}\right)\right\} \subset \Gamma\left\{d P_{m}\left(j U_{E}\right)\right\} \subset c l_{w\left(E, E^{\prime}\right)}\left\{\Gamma d f\left(j U_{E}\right)\right\}$ by [15]. Then if

$$
S=\bigcap_{j=1}^{\infty}\left(c l_{w\left(E, E^{\prime}\right)}\left\{\Gamma d f\left(j U_{E}\right)\right\} \cup \frac{U_{E^{\prime}}}{j}\right)^{\infty o}
$$

$S$ is also a weakly compact set in $E^{\prime}$ and $L_{m} \subset S$. Thus if $x \in(3 S)^{\circ}$ then $\left|P_{m}(x)\right| \leq\left[(m-1)^{m-1} /(m-1) ! m\right] 1 / 3^{m}$, so $f$ is locally bounded at 0 . Furthermore, the arguments used in Theorem 1 to prove the factorization condition (6) lead to the reflexive Banach space $\widehat{E_{\left(s^{\circ}\right)}^{\prime}}$ through which every $P_{m}$ factors. A routine calculation (see [11, Th. 2. 21]) shows that $f$ factors through $E_{(s)}^{\prime \prime}$ as a holomorphic mapping of bounded type which has a natural extension $\hat{f}$ to $\widehat{E_{\left(s^{\circ}\right)}}$. 
(2) $\Rightarrow$ (3). Since $Y$ is reflexive, the double adjoint of $T, T^{u}:\left(E^{\prime \prime}, \mu\left(E^{\prime \prime}, E^{\prime}\right)\right) \rightarrow Y$ is continuous. On the other hand, $\hat{f}$ is continuous on $Y$. Hence $f=\hat{f} \circ T$ satisfies the requirements in (3).

$(3) \Rightarrow(4)$. Since continuous polynomials are uniformly continuous on bounded sets and $f$, as a function of bounded type, is the uniform limit on bounded sets of its Taylor series expansion around 0 , it follows that $f$ is $\mu\left(E^{\prime \prime}, E^{\prime}\right)_{\left.\right|_{E}}$-uniformly continuous on bounded sets.

(4) $\Rightarrow(1)$. We begin by proving the result in the case that $f=P: E \rightarrow \mathbb{C}$, an $m$-homogeneous polynomial, $m \in \mathbb{N}$. Let $A$ be the symmetric $m$-linear mapping associated to $P$. By the polarization formula

$$
\begin{aligned}
& A\left(x_{1}, \ldots, x_{m}\right)=\frac{1}{2^{m} m !} \sum_{\epsilon_{i}= \pm 1, i=1, \ldots, m} \epsilon_{1} \ldots \epsilon_{m} P\left(\sum_{j=1}^{m} \epsilon_{j} x_{j}\right)= \\
& \frac{1}{2^{m} m !} \sum_{\epsilon_{i}= \pm 1, i=2, \ldots, m} \epsilon_{2} \ldots \epsilon_{m} P\left(\sum_{j=2}^{m} \epsilon_{j} x_{j}+x_{1}\right)-P\left(\sum_{j=2}^{m} \epsilon_{j} x_{j}-x_{1}\right) .
\end{aligned}
$$

Since $P$ is $\mu\left(E^{\prime \prime}, E^{\prime}\right)_{\mid \varepsilon}$ uniformly continuous on bounded sets, given $n \in \mathbb{N}$, there is a weakly compact set $K_{n} \subset E^{\prime}$ such that $\left|A\left(x_{1}, \ldots, x_{m}\right)\right| \leq 1$ if $\left\|x_{1}\right\| \leq n, \ldots,\left\|x_{m}\right\| \leq n$ and $x_{1} \in K_{n}^{o}$. Now let us see that $P$ is continuous for the topology on $E$ of uniform convergence on weakly compact sets in $E^{\prime}$. This will follow a very similar pattern to the proof of $(4) \Rightarrow(5)$ in Theorem 1 . Consider the weakly compact subset of $E^{\prime}$, given by $K=\bigcap_{n=1}^{\infty}\left(K_{n} \cup U_{E^{\prime}} / n\right)^{o o}$. Suppose $x \in K^{\circ}$. We will see that $|P(x)| \leq 1$, and to do this it suffices to prove that $|P(x)| \leq 1$ only for all $x \in \Gamma\left\{\cup_{n=1}^{\infty}\left(K_{n} \cup U_{E^{\prime}} / n\right)^{\circ}\right\}$. Let $x=\sum_{j=1}^{l} \alpha_{j} y_{j}$ where $y_{j} \in\left(K_{n_{j}} \cup U_{E^{\prime}} / n_{j}\right)^{o}$ and $\sum_{j=1}^{l}\left|\alpha_{j}\right| \leq 1$. For every $j, y_{j} \in\left(K_{n_{j}}\right)^{o}$ and $y_{j} \in\left(U_{E^{\prime}} / n_{j}\right)^{o}$, i.e. $\left\|y_{j}\right\| \leq n_{j}$. Then $P(x)=\sum \alpha_{j_{1}} \ldots \alpha_{j_{m}} A\left(y_{j_{1}}, \ldots, y_{j_{m}}\right)$, so it is enough to check that $\left|A\left(y_{j_{1}}, \ldots, y_{j_{m}}\right)\right| \leq 1$ for any choice of $j_{1}, \ldots, j_{m}$. We may suppose that $\left\|y_{j_{i}}\right\| \leq n_{j_{m}}$ for all $i=1, \ldots, m$. Since $y_{j_{m}} \in\left(K_{n_{j_{m}}}\right)^{o}$, we have $\left|A\left(y_{j_{1}}, \ldots, y_{j_{m}}\right)\right| \leq 1$. Thus $P$ is $\mu\left(E^{\prime \prime}, E^{\prime}\right)_{\left.\right|_{E}}$-continuous, so $A$ is also $\mu\left(E^{\prime \prime}, E^{\prime m}\right)_{\left.\right|_{E}}$-continuous. Hence $A$ is regular. Now apply Theorem 1 to see that $d P$ is weakly compact.

Finally, note that, according to Cauchy's formula, each of the Taylor polynomials of $f$ at 0 satisfies the assumption. Therefore, each has weakly compact derivative, and so we may apply Thm 3.2 of [15] to conclude that $d f$ is weakly compact.

Remark 6. The assumption that the holomorphic function $f$ be of bounded type (i.e. $f \in H_{b}(E)$ ) cannot be avoided in general. For instance, if $f: c_{0} \rightarrow \mathbb{C}$ is defined by $f\left(\left(x_{n}\right)\right)=\sum_{n=1}^{\infty} x_{n}^{n}$, then $f$ is not continuous with respect to the topology on $c_{0}$ of uniform convergence on (weakly) compact sets in $\ell_{1}$, as can be easily noticed by considering the unit basis sequence $\left\{e_{n}\right\}$ and the Banach-Dieudonne theorem. On the other hand, $d f: c_{0} \rightarrow \ell_{1}$ is weakly compact. To see this, observe that $d f\left(\left(x_{n}\right)\right)=\left(n x_{n}^{n-1}\right)$, and so each of the Taylor polynomials of $d f$ at 0 , $P_{m}[d f]\left(\left(y_{n}\right)\right)=m y_{m}^{m-1}$ is weakly compact since each takes values in a 1-dimensional subspace of $\ell_{1}$ [15]. 
The last part of this note is devoted to the relationship between the regularity and the canonical extension to the bidual of holomorphic mappings of bounded type. We refer to [4] and [6] for details about the canonical extension.

Corollary 7. If $A \in \mathcal{L}\left({ }^{m} E_{1}, \ldots, E_{m}\right)$ is regular, then its canonical extension, $\tilde{A}$, is $\mu\left(E^{\prime \prime}, E^{\prime}\right)$-continuous and regular.

Proof. Let $a_{1}, \ldots, a_{m-1} \in E$ and $z \in E^{\prime \prime}$. As in the proof of Theorem 1 , there is a net $\left\{x_{i}\right\}_{i \in I}$ in $E$ converging to $z$ in the $\mu\left(E^{\prime \prime}, E^{\prime}\right)$-topology. In particular, $\lim _{i}\left\|x_{i}\right\|_{K}=\|z\|_{K}$. Then for the canonical extension $\tilde{A}$ of $A$,

$$
\begin{gathered}
\left|\tilde{A}\left(a_{1}, \ldots, a_{m-1}, z\right)\right|=\left|\lim _{i} A\left(a_{1}, \ldots, a_{m-1}, x_{i}\right)\right| \leq \\
\lim _{i}\left\|a_{1}\right\|_{K} \ldots\left\|a_{m-1}\right\|_{K}\left\|x_{i}\right\|_{K}=\left\|a_{1}\right\|_{K} \ldots\left\|a_{m-1}\right\|_{K}\|z\|_{K} .
\end{gathered}
$$

Repeating the argument backwards, we are led to

$$
\left|\tilde{A}\left(z_{1}, \ldots, z_{m}\right)\right| \leq\left\|z_{1}\right\|_{K} \ldots\left\|z_{m}\right\|_{K} \quad\left(z_{1}, \ldots, z_{m} \in E^{\prime \prime}\right) .
$$

So $\tilde{A}$ is $\mu\left(E^{\prime \prime}, E^{\prime}\right)$-continuous and therefore $\tilde{A}$ is continuous for the topology on $E^{\prime \prime}$ of uniform convergence on weakly compact sets in $E^{\prime \prime \prime}$. Thus we can apply Theorem 1 to see that $\tilde{A}$ is regular.

We need the following lemma for the proof of our final result.

Lemma 8. Suppose that $E$ is a complex Banach space. If $M \subset H_{b}(E)$ is a relatively compact set and $d f$ is weakly compact for every $f \in M$, then $M$ is equicontinuous with respect to the topology on $E$ of uniform convergence on weakly compact sets in $E^{\prime}$.

Proof. As in the proof of Theorem 5, for every $f \in M, d f$ maps bounded sets in $E$ into relatively weakly compact sets in $E^{\prime}$. By compactness for each $n \in \mathbb{N}$ there is a finite number of elements $f_{1}, \ldots, f_{m_{n}} \in M$ such that for every $f \in M$, there is $i \in\left\{1, \ldots, m_{n}\right\}$ with $\left\|f-f_{i}\right\|_{n U_{E}+U_{E}} \leq 1 / n$. Therefore for any $x \in E$ with $\|x\| \leq n$ we have

$$
\left\|d f(x)-d f_{i}(x)\right\|=\sup _{u \in U_{E}}\left|d f(x)(u)-d f_{i}(x)(u)\right|=\sup _{u \in U_{\varepsilon}}\left|d\left(f-f_{i}\right)(x)(u)\right| \leq\left\|f-f_{i}\right\|_{n U_{E}+U_{E}} \leq \frac{1}{n}
$$

as a consequence of Cauchy's inequality. If we put $C_{n}=U_{f \in M} d f\left(n U_{E}\right)$ we have

$$
C_{n} \subset c l_{w\left(E, E^{\prime}\right)} \Gamma\left\{d f_{1}\left(n U_{E}\right) \cup \ldots \cup d f_{m_{n}}\left(n U_{E}\right)\right\}+\frac{U_{E^{\prime}}}{n} .
$$


By our assumption, the set $c l_{w\left(E, E^{\prime}\right)} \Gamma\left\{d f_{1}\left(n U_{E}\right) \cup \ldots \cup d f_{m_{n}}\left(n U_{E}\right)\right\}$ is weakly compact, and so we may again apply Grothendieck's lemma to conclude that the set

$$
C=\bigcap_{n=1}^{\infty}\left(c l_{w\left(E, E^{\prime}\right)} \Gamma\left\{C_{n}\right\} \cup \frac{U_{E^{\prime}}}{n}\right)^{o o}
$$

is weakly compact. As in Theorem 5 the functions in $M$ are uniformly bounded on $C^{o}$, from which the result follows.

Proposition 9. Suppose that $E$ is a complex Banach space and let $f \in H_{b}(E)$. Then the following are equivalent:

(1) $d f: E \rightarrow E^{\prime}$ is weakly compact.

(2) The canonical extension $\tilde{f}$ of $f$ to $E^{\prime \prime}$ is $\mu\left(E^{\prime \prime}, E^{\prime}\right)$ uniformly continuous on bounded sets.

(3) The derivative $d \tilde{f}: E^{\prime \prime} \rightarrow E^{\prime \prime \prime}$ of the canonical extension of $f$ is weakly compact.

Proof. (3) $\Rightarrow(1)$ is trivial and (2) $\Rightarrow(1)$ has been shown in the proof of (4) $\Rightarrow(1)$ of Theorem 5.

(1) $\Rightarrow$ (3). By [15], the Taylor polynomials $P_{m}$ of $f$ at 0 have weakly compact derivatives. It then follows from Corollary 7 that the canonical extensions $\widetilde{P_{m}}$ also have weakly compact derivatives. Hence, again applying [15], $\tilde{f}$ has a weakly compact derivative.

(1) $\Rightarrow(2)$. The set $M=\left\{P_{m}: m \in \mathbb{N}\right\}$ of Taylor polynomials of $f$ at zero is a relatively compact subset of $H_{b}(E)$. By Lemma 8 , there is a weakly compact set $C \subset E^{\prime}$ such that $\left|P_{m}(x)\right| \leq\|x\|_{c}^{m}$ for all $x \in E$ and $m \in \mathbb{N}$. The argument used in Corollary 7 involving the associated multilinear forms shows that $\left|P_{m}(z)\right| \leq m^{m} / m !\|z\|_{C}^{m}$ for all $z \in E^{\prime \prime}$ and $m \in \mathbb{N}$, from which it follows that the canonical extension of $f$, given by $\tilde{f}=\sum \widetilde{P_{m}}$, is locally bounded at $0 \in E^{\prime \prime}$ for the $\mu\left(E^{\prime \prime}, E^{\prime}\right)$-topology. Now, if $z_{0} \in E^{\prime \prime}$ is an arbitrary point, let $g: E^{\prime \prime} \rightarrow \mathbb{C}$ be given by $g(z)=\tilde{f}\left(z-z_{0}\right)$. Our assumption forces the Mackey continuity of the canonical extension, $\tilde{A}_{m}$, of every multilinear mapping $A_{m}$ associated to $P_{m}$, and consequently, $\widetilde{g_{1 E}}=g$. Since we know already that $d \tilde{f}$ is weakly compact, $d\left(g_{\mid \varepsilon}\right)$ is weakly compact. Thus, as we have just seen, $\widetilde{g_{\mid E}}=g$ is locally bounded at 0 , hence $\tilde{f}$ is locally bounded at $z_{0}$ for the $\mu\left(E^{\prime \prime}, E^{\prime}\right)$-topology. Therefore, $\tilde{f}$ is $\mu\left(E^{\prime \prime}, E^{\prime}\right)$ holomorphic $([11,2.2 .10])$ and since it is bounded on bounded sets, it turns out to be $\mu\left(E^{\prime \prime}, E^{\prime}\right)$ uniformly continuous on bounded sets.

Remark 10. (a) In the proof of (1) $\Rightarrow(2)$ in Proposition 9 we have shown that every holomorphic function $f: E \rightarrow \mathbb{C}$ which is uniformly continuous for the topology on $E$ of uniform convergence on weakly compact sets in $E^{\prime}$ is also continuous for this topology. Also, any of the conditions in Proposition 9 imply that, in fact, the derivative $d \tilde{f}$ has its range in $E^{\prime}$. 
(b) Our former results show that if the derivative of every polynomial is weakly compact, then the topology on $E$ of uniform convergence on weakly compact sets of $E^{\prime}$ is finer than the weak polynomial topology (i.e. convergence against polynomials). In fact, it is strictly finer in general. As an example, for the (reflexive) Tsirelson space $T^{\prime}$, all polynomials are weakly sequentially continuous (see [3]) and the unit vector basis is a weakly null sequence, hence weak polynomially null, which is not null.

\section{REFERENCES}

1. R. Alencar and K. Floret, Weak-strong continuity of multilinear mappings and the Pełczyniski-Pitt theorem, J. Math. Anal. Appl., to appear.

2. R. ARens, The adjoint of a bilinear operation, Proc. Amer. Math. Soc. 2 (1951), 839-848.

3. R. Aron, R. Alencar and S. DineEn, A reflexive space of holomorphic functions in infinitely many variables, Proc. Amer. Math. Soc. 90 (1984), 407-411.

4. R. ARon and P. Berner, A Hahn-Banach extension theorem for analytic mappings, Bull. Soc. Math. France 106 (1978), 3-24.

5. R. Aron, S. Y. Chor and J. L. G. Llavona, Estimates by polynomials, Bull. Austral. Math. Soc. 52 (1995), 475-486.

6. R. Aron, B. Cole and T. Gamelin, Spectra of algebras of analytic functions on a Banach space, J. Reine Angew. Math. 415 (1991), 51-93.

7. R. Aron and S. Dineen, Q-reflexive Banach Spaces, Rocky Mountain J. Math., to appear.

8. R. Aron, P. Galindo, D. García and M. Maestre, Regularity and Algebras of Analytic Functions in Infinite Dimensions, Trans. Amer. Math. Soc. 348 (1996), 543-559.

9. R. Aron, C. Herves and M. Valdivia, Weakly continuous mappings on Banach spaces, J. Funct. Anal. 52 (1983), 189-204.

10. J. Diestel, Sequences and series in Banach spaces (Graduate Texts in Math. 92, SpringerVerlag, New York, 1984).

11. S. Dineen, Complex Analysis in Locally Convex Spaces (Math. Studies 57, North-Holland, Amsterdam, 1981).

12. J. FARMer, Polynomial Reflexivity in Banach Spaces. Israel J. Math. 87 (1994), 257-273.

13. H. JARCHOW, Locally Convex Spaces (Teubner, 1981).

14. A. PęCZYǸSKI, On weakly compact polynomial operators with Dunford-Pettis property, Bull. Acad. Polonaise Sc., 11 (1963), 371-378.

15. R. Ryan, Weakly compact holomorphic mappings on Banach Spaces. Pacific J. Math. 131 (1988), 179-190.

16. E. SAAB and P. SAAB, Extensions of some classes of operators and applications, Rocky Mountain J. Math. 23 (1993), 319-337.

17. E. Toma, Aplicações holomorfas e polinómios $\tau$-continuos (Thesis, Univ. Federal do Rio de Janeiro, 1993). 
18. A. Ülger, Weakly compact bilinear forms and Arens regularity, Proc. Amer. Math. Soc. 101 (1987), 697-704.

Mathematics Department

Kent STATE University

KENT

OHIO 44242

U.S.A.

E-mail: aron@mcs.kent.edu
Departamento de Análisis Matemático

UNIVERSIDAD DE VALENCIA

DOCTOR MOLINER 50

46100 BURJASOT (VALENCIA)

SPAIN

E-mail: galindo@uv.es 